\title{
Surgical Stabilisation of the Cervical Spine: A Comparative Analysis of Anterior and Posterior Spine Fusions
}

\author{
Daniel A. Capen, M.D., Russell W. Nelson, M.D., Jack Zigler, M.D., \\ Robert L. Waters, M.D., Douglas E. Garland, M.D. \\ Channel Islands Orthopedic Medical Group, 300 South 'A' St. 105, Oxnard, \\ CA 93030, U.S.A.
}

\section{Introduction}

Advances in the treatment of cervical spine trauma have occurred since Hadra initially internally fixed an unstable cervical spine in 1891. Methods of dealing with complex neurological injury have been foremost in the mind of the treating surgeon. Open reduction and early spine fusion has become a cornerstone of treatment to promote alignment and to protect against further neurologic damage. Internal fixation and reduction can be done by either an anterior or a posterior approach, and there are proponents for both these approaches. The purpose of this paper is to review a large number of stabilisation procedures to evaluate perioperative morbidity, late complications and the efficacy of stabilisation.

\section{Patients and methods}

We reviewed 212 patients who underwent 222 operations. We studied postoperative radiographs, and evaluated patients at follow-up for radiographic evidence of healing and for functional status.

There were 179 men and 43 women, with an age range from 10 to 65 years. The average age was 26.7 years and the median age was 20.5 years. The most frequent causes of trauma were motor vehicle accidents and water sports (Table I). The skeletal level of injury most commonly involved was the lower cervical spine. Neurologic involvement was complete in 96 patients and incomplete in 99 patients. Seventeen patients were neurologically normal at the time of sur-

Table I Etiology of Injury in Patients with Surgical Stabilisation

\begin{tabular}{lrr}
\multicolumn{1}{c}{ Etiology } & Patients & Per cent \\
\hline MVA & 102 & $48 \cdot 1$ \\
Swim and dive & 61 & $28 \cdot 7$ \\
Falls & 19 & $8 \cdot 9$ \\
Sports & 18 & $8 \cdot 4$ \\
Pedestrian & 8 & $3 \cdot 7$ \\
Other & 4 & $1 \cdot 8$ \\
\hline
\end{tabular}


gery. The extent of neurologic injury did not exclude the use of either surgical approach, with 88 patients undergoing anterior fusion, 114 undergoing posterior fusion and 10 underwent both procedures.

Of those who had anterior cervical fusions, 37 had complete lesions, 47 incomplete and 4 were normal. In the posterior surgical group 57 were complete, 44 incomplete and 13 were normal, and in the combined surgical group 2 were complete, 8 incomplete.

Nineteen of the patients in this study did not have a fracture or dislocation, but there was significant cervical spondylosis with large posterior osteophytes impinging anteriorly on the spinal cord. Injury forces, most frequently hyperextension, caused a central cord syndrome. These patients were treated by anterior decompression and fusion.

Of the 114 posterior spine fusions, 98 were followed up from 5 months to 13 years. Sixteen were lost to follow-up. The average follow-up period was 4 years and 2 months. Eighty-six patients had more than 2 years' follow-up. Fifty-nine of the 88 anterior spine fusion cases were followed from 3 months to 12 years. The average follow-up period was 3 years and 8 months with 51 having more than 2 years' follow-up. Nine of the 10 patients treated by combined fusion were followed an average of 1 year and 10 months with 5 patients having 2 years of follow-up. All of the patients in this study had at least a 3 month follow-up in the postoperative period (Table II).

Table II Follow up for Surgical Patients

\begin{tabular}{lcc}
\hline & Two year follow-up & Average follow-up \\
\hline Posterior fusion & 86 & 4 years 2 months \\
Anterior fusion & 51 & 3 years 8 months \\
\hline
\end{tabular}

\section{Surgery}

In this study, all but three of the patients in the anterior spine fusion group underwent a Smith-Robinson anterior approach with vertebrectomy of the fractured segment followed by a fibular strut graft. The other three patients had a tricortical iliac crest graft (Fig. 1).

The posterior spine fusion group of patients underwent a Rogers-type posterior wiring and fusion utilising an 18 gauge wire passed through the spinous process above the level of instability and around the spinous process below the level of instability. This encompassed three levels in most cases, but on occasion it was necessary to fuse more than 3 levels because of fractured vertebral bodies (Fig. 2).

\section{Results}

Surgical complications: No perioperative mortality occurred with any of the cervical spine stabilisation procedures. Surgical blood loss was similar in these procedures averaging $639 \mathrm{cc}$ for the anterior fusion group, $651 \mathrm{cc}$ for the posterior fusion group and $725 \mathrm{cc}$ in the combined surgical group. Local complications and systemic complications were noted at relatively equal frequency 

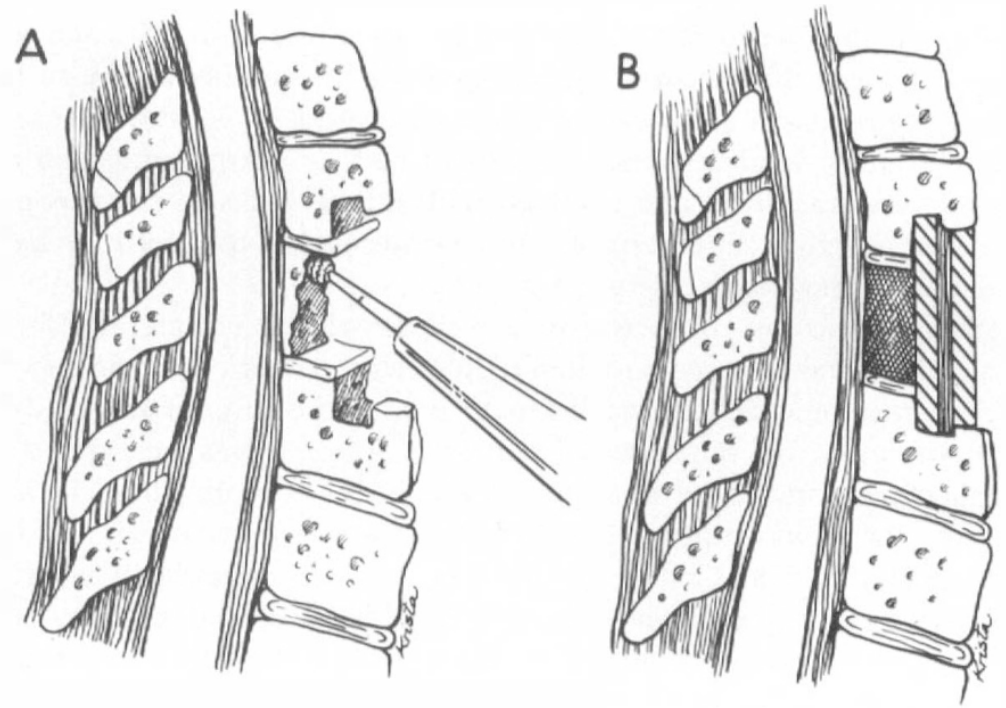

Figure 1a, 1b Anterior bone and disc material are removed and tricortical iliac crest or fibular strut grafting is performed to achieve stability.

A

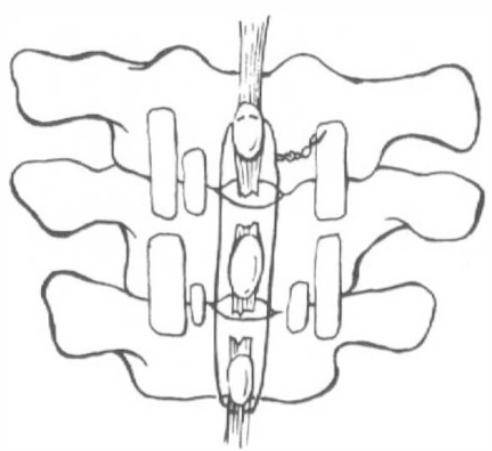

B

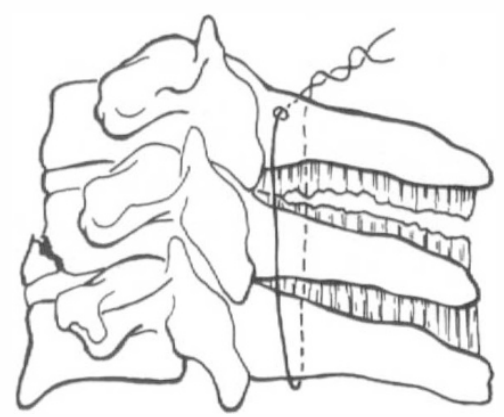

Figure 2a, 2b A single loop of 18 gauge wire is passed through the superior portion of the spinous process above the lesion and around the process below. This is followed by limited onlay of corticocancellous strips to achieve fusion.

with the exception of a significant number of dysphagia and dysphonia problems in eight of the cases of anterior fusion $(9 \%)$. These resolved spontaneously but resulted in loss of rehabilitation time and delay in rehabilitation because of the inability to tolerate fluids, inability to swallow and inability to communicate appropriately. Deep venous thrombosis, pulmonary complications and wound complications were evenly distributed and were un-related to the type of surgery.

Post surgical neurologic deficit in patients undergoing anterior surgical stabilisation occurred in 4 of 88 cases, $(4.5 \%)$. These postoperative deficits were mixed motor and sensory changes secondary to surgical manipulation of 
the spinal cord and nerve roots. Root compromise was noted in two patients with temporary loss of one wrist muscle grade in the immediate post surgical period. One patient with a central cord lesion suffered 2 weeks of diffuse upper and lower extremity weakness and one patient had C6 dermatome sensory loss. All changes were transitory and resolved within 6-8 weeks to the preoperative status without surgical intervention. It was necessary to continue halo immobilisation until neurologic return was noted.

Severe neurologic loss occurred in a patient with a unilateral C5-6 facet dislocation which was reduced and then treated with posterior wiring and fusion. A transient neurologic deficit had been present at the time of injury, but this resolved completely by the time of surgery. Surgery was performed under general anaesthesia and there were no intraoperative complications. The initial postoperative status was normal, but 18 hours post surgery the patient became totally quadriplegic at the $\mathrm{C} 5$ level. Myelography demonstrated an anterior soft tissue lesion and re-exploration with wire removal and halo traction failed to restore function. Subsequent anterior decompression revealed disc fragments and some bone fragments causing cord compression. This was the only neurologic complication in the 114 patients included in this study.

No patient undergoing combined anterior and posterior surgical fusion had any neurologic loss. The overall incidence of permanent neurologic impairment for all stabilisation procedures was one out of 222 procedures $\left(0.4^{\circ}{ }_{0}\right)$. The average total blood loss was less than $130 \mathrm{cc}$, and was slightly more for those who had combined anterior and posterior fusions.

Techniques have improved and because we now do limited dissection posteriorly, the average blood loss has been reduced to $300 \mathrm{cc}$ in recent cases.

Complications for this combined surgical group were one patient with deep venous thrombosis, one with dysphagia for 4 weeks and one patient who had cardiac arrhythmia after the second stage of the procedure. All these complications responded to treatment and the patients completed their rehabilitation programme.

\section{Postoperative follow-up}

\section{Anterior cervical fusion}

In the 59 patients followed up after undergoing anterior cervical spine fusion, there were several complications. Direct dislodgement of the fibular strut graft occurred in the immediate postoperative period in six of the cases $\left(10^{\circ} \%\right)$. All six cases of graft dislodgement required surgical replacement. In all six cases, there was definitive widening of the posterior elements as seen in the postoperative radiographs to suggest posterior ligamentous instability. Preoperative radiographic examination also demonstrated subtle facet or interspinous widening.

Thirty-two of the 59 patients had a loss of the initial reduction at the time of surgery with the development of progressive anterior kyphotic angulation (Fig. 3). Twenty-one of the patients had no measurable angle on follow-up evaluation. Six patients had an angulation of up to 11 degrees. Sixteen other patients had kyphotic angulation of between 12 and 20 degrees an 14 patients had kyphotic 

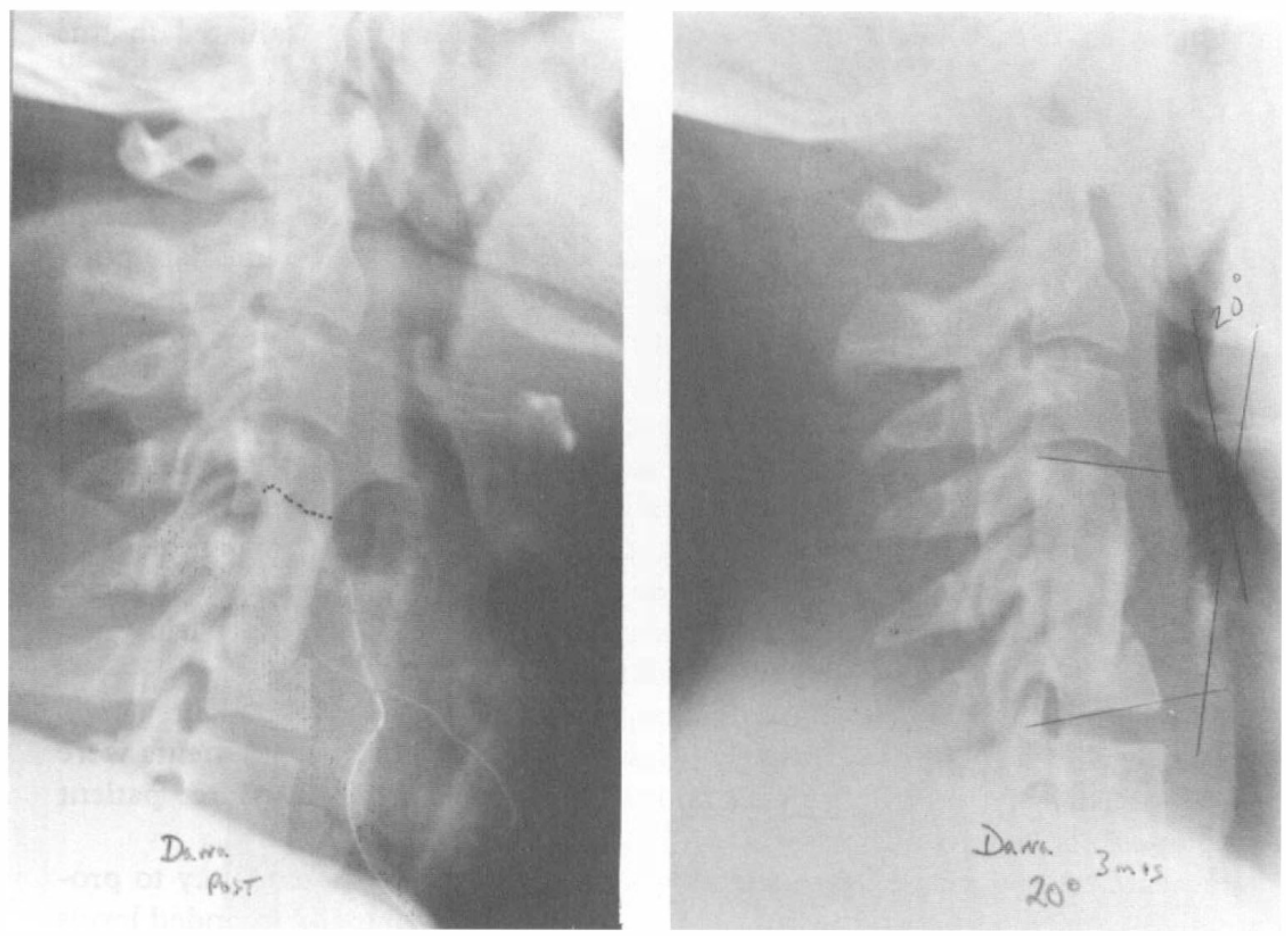

Figure 3a, 3b Initial post surgical radiograph shows good reduction but at 3 months there is settling of the graft and early kyphosis and posterior ligament widening.

angulation of between 21 and 30 degrees. Two patients had an angulation greater than 30 degrees. The average angulation of the patients was 22 degrees. Deformity progression was measurable between the immediate postoperative stages until solid fusion was obtained. No measurable progression was noted beyond 16 months after surgery. Lateral radiographs demonstrated progressive settling of the corticofibular strut into the cancellous vertebral bodies above and below the vertebrectomised segment. A progressive widening of the posterior facets and interspinous processes to suggest ligamentous damage was noted with the development of the kyphotic deformity in all cases.

In the 19 patients with hyperextension traumatic spondylitic myelopathy, no demonstrable pre or postsurgical posterior ligamentous instability occurred. These patients were all treated successfully by anterior decompression and fusion. Fifteen patients had undergone laminectomy prior to transfer from another facility which clearly compromised posterior ligamentous stability. Twenty-three of the 36 remaining patients treated by anterior stabilisation developed a significant deformity for $63 \cdot 8^{\circ}$ o failure of the procedure to maintain alignment in cases initially evaluated as suitable for primary anterior arthrodesis.

The development of spondylitic lipping and spurring and uncinate process hypertrophy occurred in 36 of the 59 patients studied on long term X-ray followup. This occurred at the disc levels above and below the fusion and represents the development of degenerative changes in $64 \%$ of these patients. Sympto- 
matology and late effects on nerve root function were not evaluated in this study.

\section{Posterior cervical spine fusion}

Ninety-eight of the 114 patients could be followed up. Failure of wire fixation was noted in two patients. One wire breakage occurred in the immediate postoperative period and this patient returned with a non-union. There was a second wire breakage that occurred that did not result in non-union and was treated in a Philadelphia collar with no loss of reduction.

Wire pullout through the spinous process above the level of injury was noted in two cases. This spinous process was fractured in one of the cases. Failures of fixation were noted in the early postoperative period and were treated with semirigid cervical collar immobilisation and observation. No loss of alignment or compromise of fusion was noted. None of the patients developed posterior displacement or angulation of greater than 11 degrees. Once the wiring and fusion had been performed, no loss of reduction occurred.

Radiographic evidence of degeneration occurred in two of 98 patients $(2 \%)$. These changes developed above and below the fusion mass. Both patients were over 40 . Changes were noted at 2 and $3 \frac{1}{2}$ years respectively. Neither patient developed noted pain.

Patients treated by posterior wiring and fusion displayed a tendency to progressive extension of posterior fusion mass beyond the originally intended levels of fusion (Fig. 4). This extension became evident 6 and 12 months after surgery
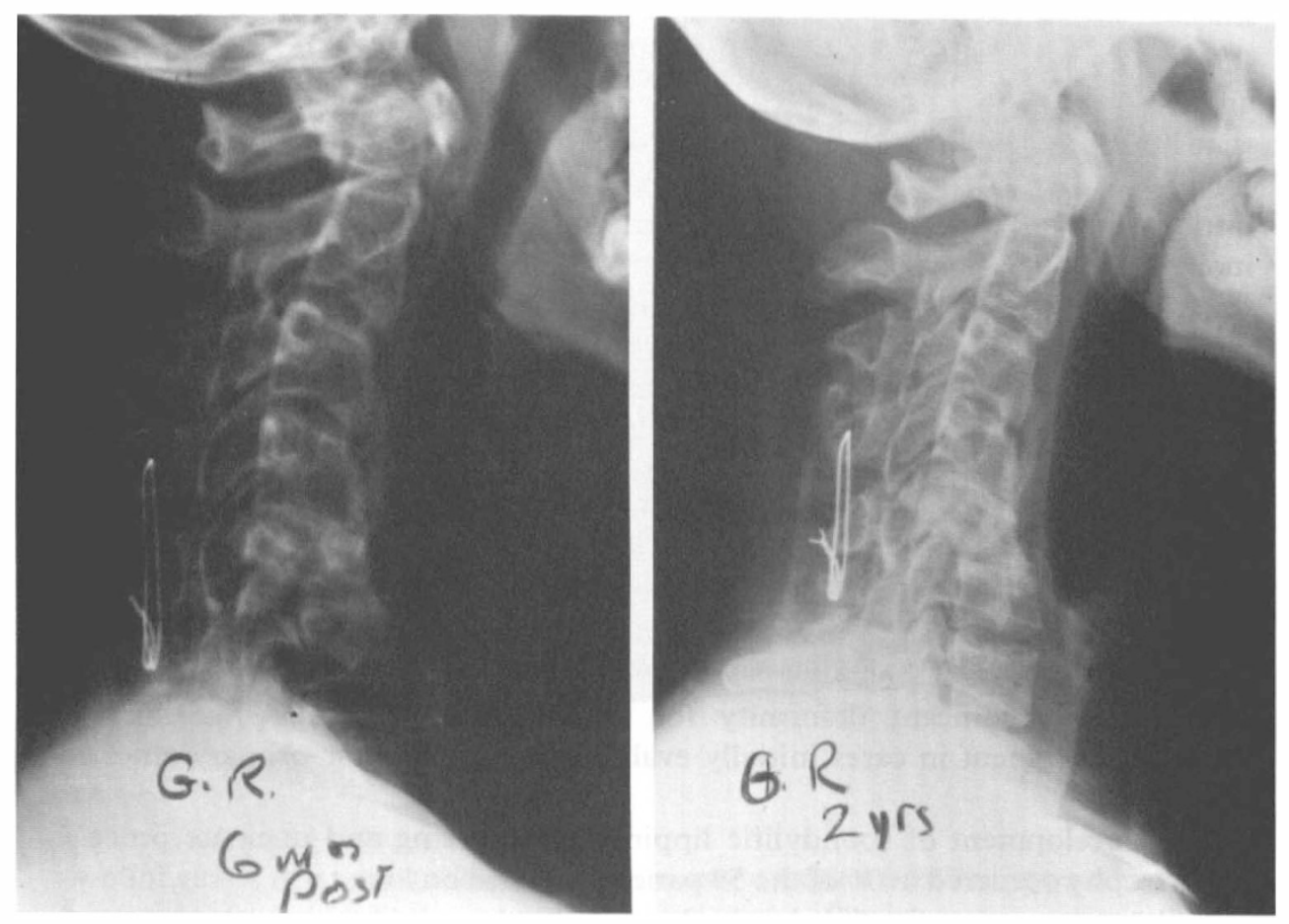

Figure 4a, $4 \mathbf{b}$ The post surgical extension of fusion mass is noted at 6 months and more clearly at 2 years both above and below the original three level fusion. 
and progressed to the point where 73 of the 98 patients $(74.4 \%)$ had extension of fusion mass at least one level above or below the originally intended level of stabilisation. Thirty-six patients had extension to more than one level and 13 patients had more than two levels.

A review of the operative dictations revealed a direct correlation between the extent of exposure of the posterior elements and extension of the fusion mass in 30 patients. The long term effects of extension of the fusion mass was not included in this study. The previous tendency towards wide exposure of the entire fracture site and all spinous processes and all posterior elements led to application of graft material over a wider area, thus leading to the extension of the fusion.

\section{Discussion}

With the advances in techniques of treating quadriplegia, it is imperative to appreciate that these patients are living to an almost normal life span. These injuries occur primarily in young people. Surgical choice and the late sequelae of the surgery must be most carefully considered.

The literature strongly supports the utilisation of both anterior and posterior cervical stabilisation procedures for the treatment of cervical spine instability. From the study that we have made, it is apparent that there are definite indications for both types of procedures. There are also some cautions and considerations that must be made when utilising these surgical approaches.

In 1976 Stauffer and Rhodes advocated that the surgical approach be tailored to the location and type of instability. We feel that there are definite indications for both anterior and posterior fusion. However, initial radiographs can be misleading. The recently described classification by Ben Allen informs us of the existence of significant posterior ligamentous instability, even when the initial injury radiographs do not reflect a gross and obvious fracture or dislocation. It is also important to consider that with improvement in initial emergency care and transfer, a significantly dislocated cervical spine can frequently be reduced, either spontaneously or with positioning by emergency technicians prior to any radiographic examination. A misleading sense of security can be present when the radiograph obviously shows vertebral body fracture without definitive dislocation. The extent of soft tissue injury cannot always be accurately assessed from radiographs and CT and MRI scans may be required.

Several series have noted that skeletal complications have occurred with regularity in using anterior cervical stabilisation procedures. Graft dislodgement and kyphotic angulation have been alluded to previously by Cloward and other authors. In our series, this was found to be a very significant complication frequently resulting in loss of reduction and graft dislodgement requiring a second operation.

Because of the literature and our experience, we recommend that unless direct spinal cord decompression is required in patients with deteriorating neurologic function or in a patient with an incomplete lesion who has stabilised and plateaued in recovery, the stabilisation of choice is a posterior wiring and fusion. This prevents the risk of development of significant kyphotic deformity. It also prevents the risk of graft dislodgement that requires additional surgery and 
delays rehabilitation. Anterior decompression and fusion for complete neurologic injuries is advocated in some centres for facilitation of root recovery but has not been statistically proven to be a necessary procedure for these patients.

The clear superiority of posterior wiring and fusion to maintain fracture reduction and alignment has been demonstrated in our series. The surgeon is advised to perform limited dissection and limit bone graft application to help to prevent unplanned fusion extension. The procedure permits halo-free postoperative rehabilitation. The operation is recommended to treat the vast majority of traumatic lesions of the skeletal structure and ligaments in the cervical spine trauma.

From the results of this large series of anterior and posterior cervical spine fusion, the authors recommend that the surgeon consider the following for acute fractures and fracture dislocations of the cervical spine:

1. In patients with spinal cord injury secondary to cervical spine trauma, expect a high incidence of significant structural ligamentous disruption in both the anterior and posterior columns. The absence of widening of the posterior vertebral elements on preoperative radiographs is not absolute evidence that ligamentous stability is present.

2. Posterior wiring and fusion is an excellent means of spine stabilisation that does not depend on the integrity of the posterior ligamentous structures. This procedure allows early postoperative mobilisation without a halo vest and provides for long-term maintenance of fracture reduction.

3. The use of anterior cervical spine fusion alone as a primary means of stabilisation may be complicated by progressive loss of reduction or graft dislodgement in the early postoperative period. The authors recommend that anterior surgery be reserved for cases requiring decompression to improve neurologic function or where extensive laminectomy has made posterior surgery inadvisable.

4. For patients requiring anterior decompression of the cervical spinal cord, either prolonged immobilisation in a halo until fusion is demonstrated or additional posterior wiring and fusion should be undertaken to prevent graft dislodgement and progressive loss of alignment.

5. Every effort should be made to limit the amount of posterior dissection in the performance of posterior wiring and fusion. Also, a small amount of corticocancellous graft inserted in the area of the intended fusion is sufficient to provide for stabilization of the spine. This will help to prevent unplanned extension of the fusion mass beyond the level of wiring.

\section{References}

Allen GL, Ferguson RL, Lehmann TR, et al. 1982 A mechanistic classification of closed indirect fractures and dislocations of the lower cervical spine. Spine 7(1):1-27.

BAILEY RW, BADGLEY CE 1960 Stabilisation of the cervical spine by anterior fusion. fournal of Bone and foint Surgery 42A(4):565.

Bell GD, BAILEY SI 1977 Anterior cervical fusion for trauma. Clinical Orthopaedics 128:155.

ClowARD RB 1961 Treatment of acute fractures and fracture dislocations of the cervical spine by vertebral body fusion. A report of eleven cases. Fournal of Neurosurgery 18:201.

HADRA BE 1891 Wiring of the spinous process in injury and Pott's disease. Transactions of the American Orthopedic Association 4:206.

HUNTER LY, BRAUNSTEIN EM, BAILEY RW 1980 Radiographic changes following anterior cervical fusion. Spine 5(5):399. 
PIERCE DS 1969 Spinal cord injury with anterior decompression, fusion, stabilisation and early rehabilitation. Fournal of Bone and foint Surgery 51 A:1675.

RoBINSON RA 1964 Anterior and posterior cervical spine fusions. Clinical Orthopaedics 35:34. RoBINSON RA, SMITH GW 1958 The treatment of cervical spine disorders by removal of the intervertebral disc and interbody fusion. Fournal of Bone and foint Surgery 40A:607.

ROGERS WA 1942 Treatment of fracture dislocation of the cervical spine. Fournal of Bone and foint Surgery 24:295.

ROGERS WA 1957 Fractures and dislocations of the cervical spine, and end-result study. fournal of Bone and foint Surgery 39A:2.

SHIELDS CS, STAUFFER ES 1976 Late instability in cervical spine fractures secondary to laminectomy. Clinical Orthopaedics 119:144.

STAUFFER ES, KELLY EG 1977 Fracture dislocations of the cervical spine. Fournal of Bone and foint Surgery 59A:45.

STAUFFER ES, RHOADES MD 1976 Surgical stabilisation of the cervical spine after trauma. Archives of Surgery 111:652.

VAN Peteghem PK, Schweigel JF 1979 The fractured cervical spine rendered unstable by anterior cervical fusion. Fournal of Trauma 19(2):110. 